\title{
Human response in gazing at a moving figure
}

\author{
Takada, H., Sato, Y., Shibata, H. \\ Yokohama National University, Dept. of Mechanical Eng. and Material Science, Yokohama, Japan
}

\begin{abstract}
This paper deals with the human body vibration in gazing at a moving figure. When an automatic control breaks down in an atomic power plant, and man has to operate manually, a severe accident may occur if man operates by mistake In this paper, movements of man's center of gravity were measured when he was gazing at a vibrating picture made by a personal computer in a darkroom. As a result, man's center of gravity moves in his own resonance frequency.
\end{abstract}

\section{INTRODUCTION}

Some people work under severe conditions like a moving environment or with handling a vibrating tool. A worker directly receives vibrations from a vibrating tool or indirectly receives vibrations because objects which he is gazing at is vibrating. Such a bad labor environment is not only a problem for workers but also the worker may operate by mistake. This fact is concerned with a human operating error in an emergency because man acts reflexly by an influence of informations he gets. In an atomic power plant when an automatic control breaks down because of an earthquake and man has to operate manually, a severe accident may occur if man operates by mistake. Man gets a great part of informations by eyes, but the information by man's eyes may not be true. For example, when a man in a train is gazing at another starting train, he thinks his train is starting, and another man thinks another train is going. It is very important which imagination he has. If he thinks another train begins to start when his train is starting, he loses his balance. Even in the opposite case; if he thinks another train is going when his train begins to start, he can also lose his balance. There are a lot of papers on body vibrations in shaking the human body. There are also some papers (M.J.Griffin 1975, 1976, A.J.Benson and G.R.Barnes 1978) on eye movements or vision, but few papers on human body vibration (K.Taguchi et al. 1981) in gazing at a moving object. In this paper, we consider how man swings reflexly when he is gazing at a moving picture. Movements of man's center of gravity ( C.G. ) were measured when he standing on a force plate in a darkroom is gazing at a swinging picture made by a personal computer. As a parameter, the period and movement pattern of the vibrating picture were considered. The subject's own resonance frequency and the phase difference between the picture and his C.G. were calculated by cross-relation function. This study makes the operation efficiency and influences on a human body under the bad labor environment clear. The results obtained from experiments on a human body 


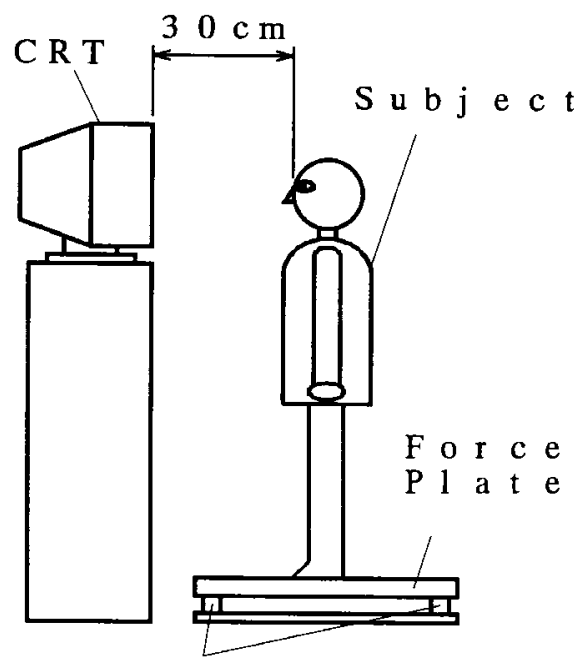

L o a d

t $r$ a $n$ s d u c e r

Fig.1 Experimental apparatus

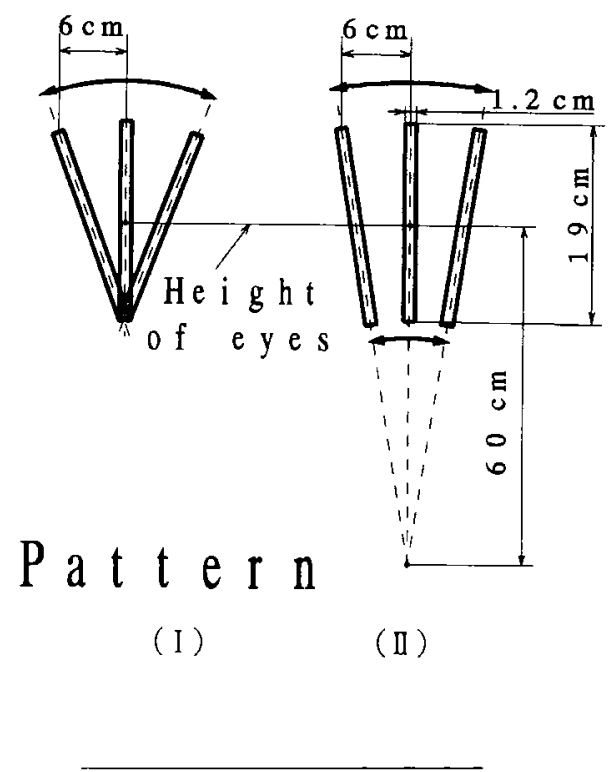

Fig.2 Moving picture

determine parameters of human vibrating model, by which a good environment or a good man-machine interface is designed.

\section{EXPERIMENTS}

The positions of man's C.G. were measured with four load transducers under the force plate in a dark room as shown in Fig.1. And the phase difference between C.G. position and the moving picture was also measured. The rectangular picture, which is $12 \mathrm{~mm}$ wide and $190 \mathrm{~mm}$ long, swings with the vertical position as a center in a 21 inches display. Two patterns were tried; pattern I and pattern II as shown in Fig. 2. The picture swings at the frequency range of $0.1 \sim 1 \mathrm{~Hz}$. In the pattern I the picture swings with its bottom as a center of rotation, and in the pattern II it swings with the height of man's waist as a center of rotation. The subject's eyes are $30 \mathrm{~cm}$ away from the picture and the both heights are the same. As a distance between feet, 0 and $15 \mathrm{~cm}$ were chosen. One trial was continued in the period of 80 seconds. Six graduate students were examined to reduce differences between individuals.

\section{EXPERIMENTAL RESULTS AND DISCUSSION}

\subsection{C.G. movements in gazing at a moving picture}

C.G. movements in gazing at a vertical stationary bar in a dark room in the period of 80 seconds were compared with those in gazing at one vibrating at $0.1 \mathrm{~Hz}$. As shown in Fig.3, the result indicates man can more stably stand in gazing at a vertical stationary bar than in gazing at a moving one. And judging from this figure, man stands more unstably at $0.25 \mathrm{~Hz}$ and less in the latter case than in the former case. The locus of C.G. projected on the force plate is shown in Fig.4. There is drawn the feet position, too. The C.G. swings not only right and left but also back and forth. 


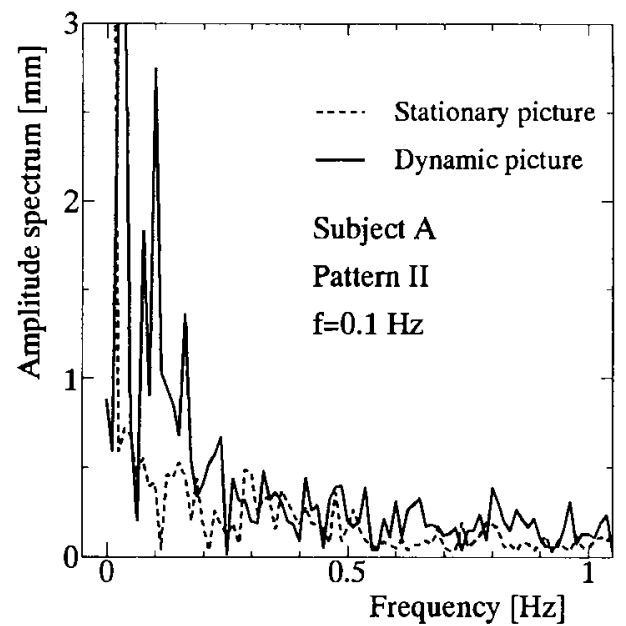

Fig.3 Difference of C.G. movements between stationary picture and dynamic one

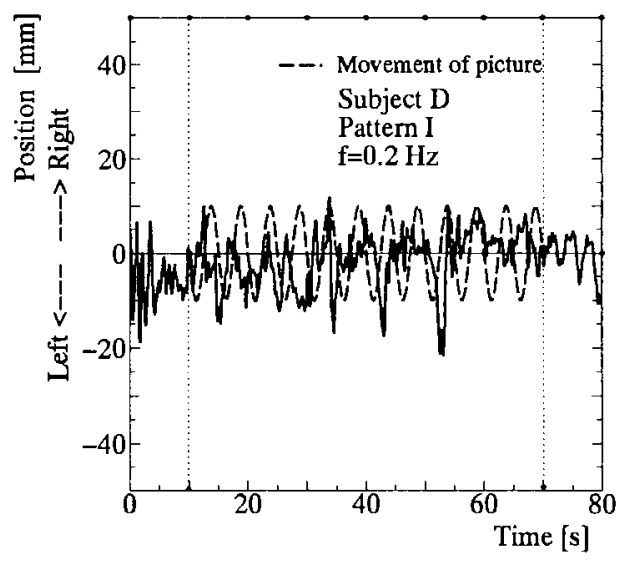

Fig.5 (a) Movements of center of gravity

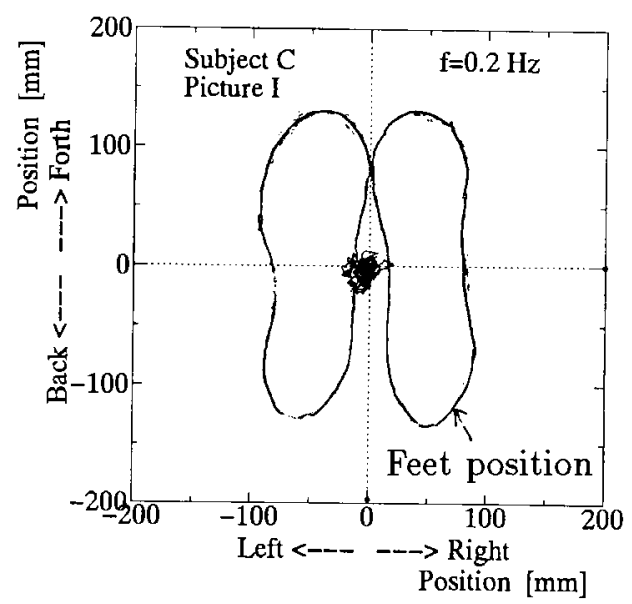

Fig.4 Locus of center of gravity

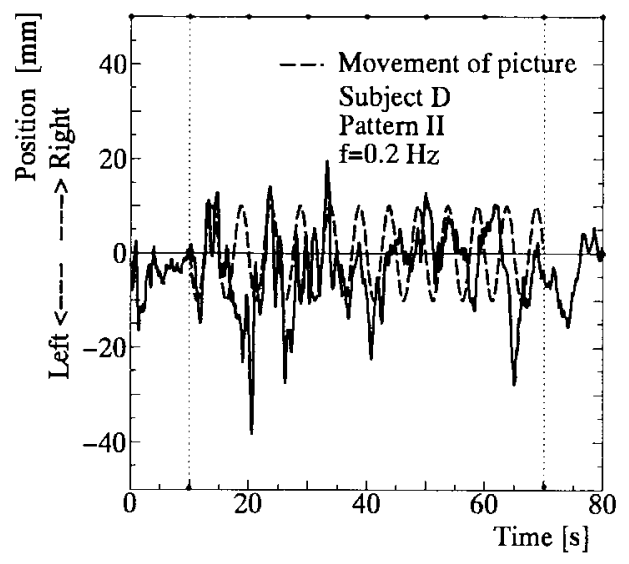

Fig.5(b) Movements of center of gravity

Man swings with one point of his body, for example, a neck, a waist or a pair of heels, as a center of rotation in swinging right and left. Two patterns shown in Fig.2 were tried to determine which part of man's body was the center of rotation. Here, in the first period of 10 seconds and in the last period of 10 seconds, the picture is stationary, and in the other period of 60 seconds it swings. As shown in Figs.5(a) and (b), man swings more violently in gazing at the pattern II than in gazing at the pattern I. This means man swings with his waist as a center of rotation. In this study we use the pattern II.

The stability of standing position is due to the distance between a subject's feet, too. Figure 6 shows differences of C.G. movement with the distance between feet as a parameter. Figure 6(a) shows the C.G. movements when a subject stands with the distance $0 \mathrm{~cm}$ between feet, and 6(b) shows those when he stands with the distance of $15 \mathrm{~cm}$. From this results, the amount of the movement in the former case is larger 


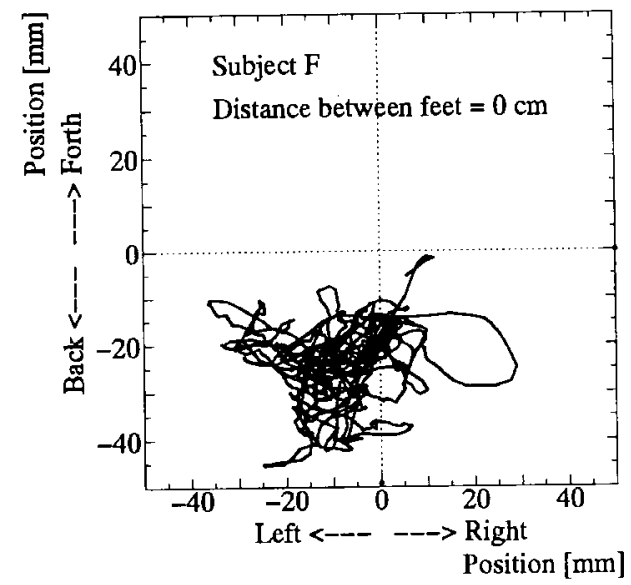

Fig.6 (a) Locus of center of gravity

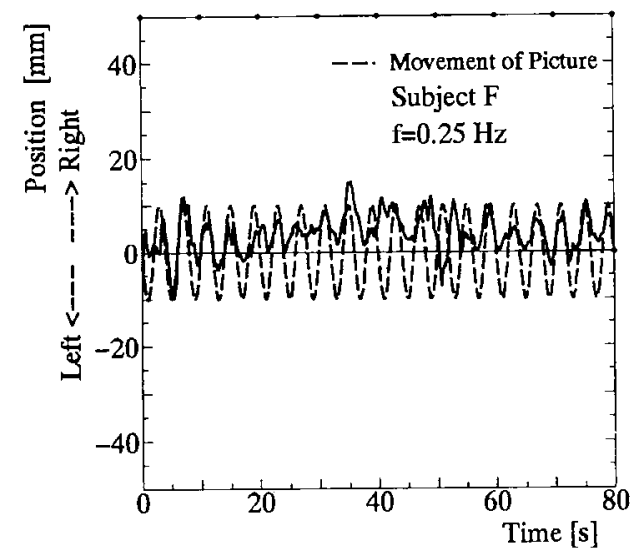

Fig.7(a) Movements of center of gravity

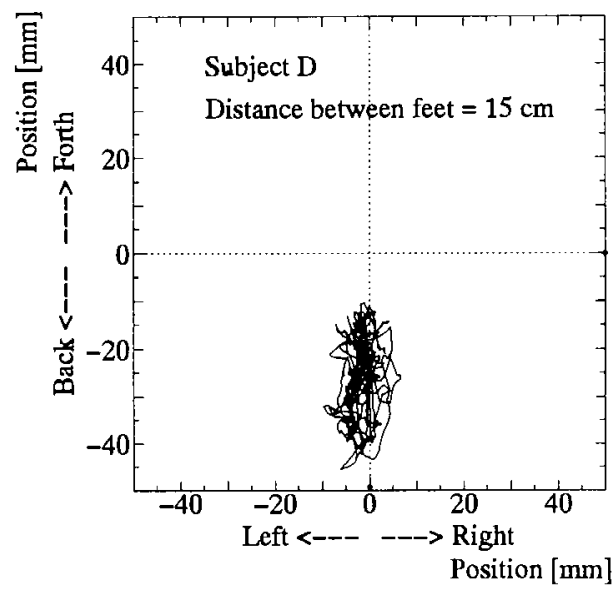

Fig.6 (b) Locus of center of gravity

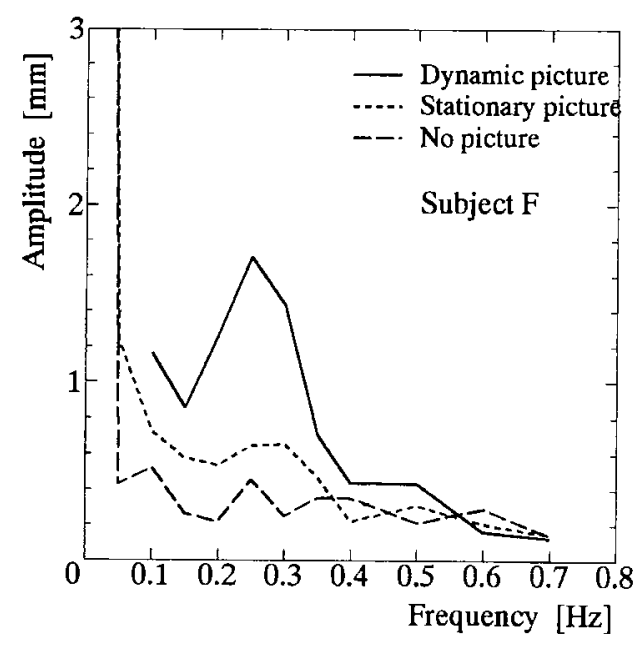

Fig.7(b) Frequency response

than that in the latter case, but it didn't go well because the data in the former case have a lot of noise. Therefore, in this study the distance of $15 \mathrm{~cm}$ were used.

\subsection{Frequency response and phase difference}

The amount of C.G. movements like a vibration amplitude is due to the frequency at which the picture swings. From Fig.7(a), it looks man sometimes tunes in the same period as the movement of picture, but he does not always tune with it. The frequency response of one subject is shown in Fig.7(b), which indicates that he has a resonance frequency $0.25 \mathrm{~Hz}$. In addition, frequency responses of six subjects are shown in Fig.8, which indicates that they have their own resonance frequencies in the range of $0.1 \sim 0.3 \mathrm{~Hz}$. The phase difference between a C.G. swing and a picture swing is shown in Fig.9. The values are the mean of 3 trials and the values $\sigma$ are its deviations. This results indicate that the phase difference at the resonance 


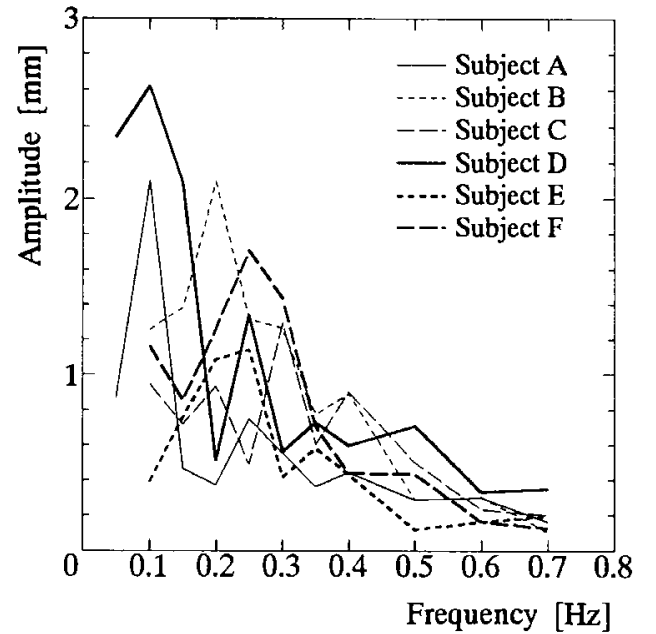

Fig.8 Frequency response

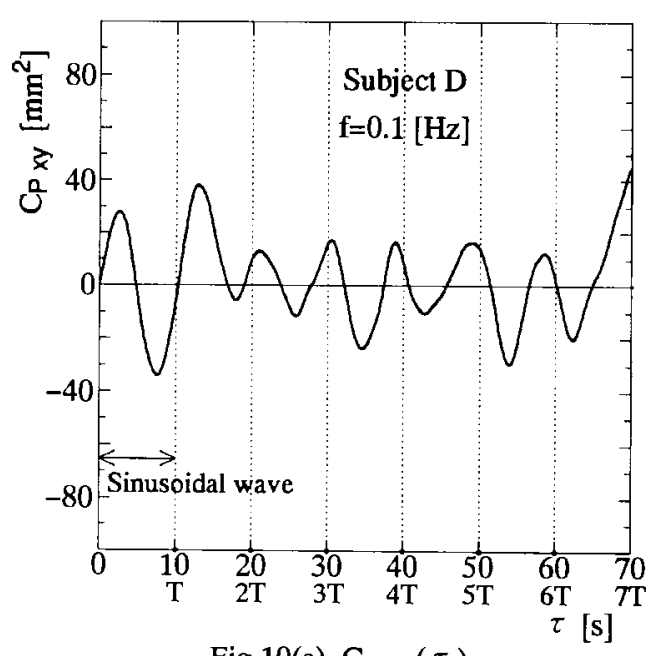

Fig.10(a) $C_{p x y}(\tau)$

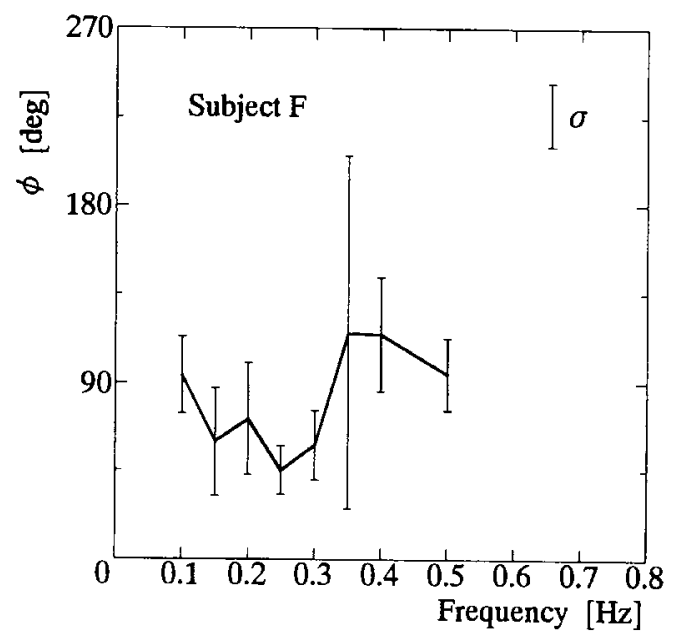

Fig.9 Phase difference

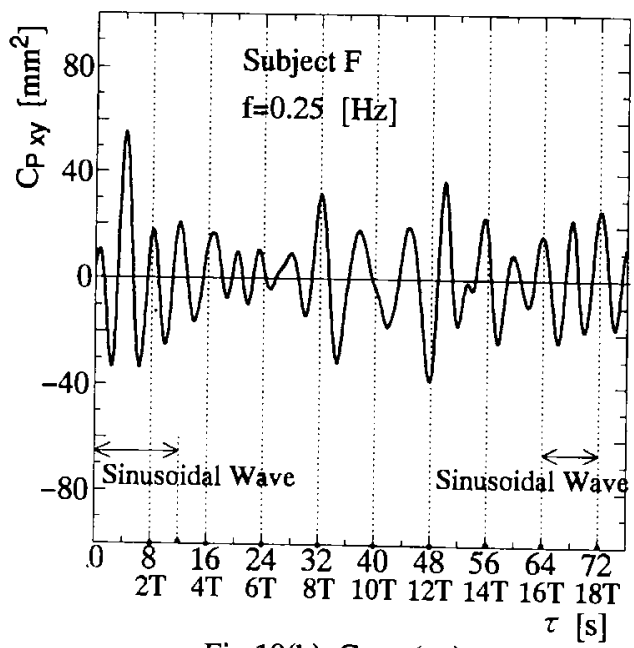

Fig.10(b) $C_{p x y}(\tau)$

frequency $0.25 \mathrm{~Hz}$ is the smallest and its deviation is the smallest, too. This means man can stand the most stably at his own resonance frequency and the results of the examination are reproducible. The cross-relation function is calculated to obtain what time of the examination man swings in the same period. Figure 10(a) shows the calculation results. The frequency $0.1 \mathrm{~Hz}$ is the subject's resonance frequency. This figure indicates that in the first period of the examination he swings at the same frequency as the moving picture swings but gradually swings delay. The results of another subject are shown in Fig. 10(b). The frequency $0.25 \mathrm{~Hz}$ is his resonance frequency. The figure indicates that in the first and a half period he swings at the same frequency as the moving picture swings but gradually swings delay, and at the last period he swings simultaneously again. Even if man swings with his resonance frequency, he does not continue to swing in the frequency, but he sometimes swings at the frequency of the moving picture and sometimes swings at another frequency. 


\section{CONCLUSIONS}

Movements of man's center of gravity were measured when he was gazing at a vibrating picture in a darkroom. The conclusions are as follows;

1. Man's C.G. movements in gazing at a vertical stationary bar is smaller than those in gazing at a moving one.

2. Even when man is gazing at a picture moving right and left, he swings not only right and left but also back and forth.

3. Man swings with his waist as a center of rotation.

4. Man sometimes tunes at the same frequency as the movement of picture swings, but he does not always tune with it. Six subjects have their own resonance frequencies and they swing in the range of $0.1 \sim 0.3 \mathrm{~Hz}$.

5. As for the phase difference between a C.G. swing and a picture swing, it is the smallest at the resonance frequency and its deviation is the smallest, too. Therefore, man can stand most stably at his own resonance frequency and the results of the examination are reproducible.

\section{REFERENCES}

1. M.J.Griffin, Levels of Whole-Body.Vibration Affecting Human Vision, Aviation, Space, and Environmental Medicine, August, 1975, 1033-1040.

2. M.J.Griffin, Eye Motion during Whole-Body Vertical Vibration, Human Factors, $1976,18(6), 601-606$.

3. A.J.Benson and G.R.Barnes, Vision during Angular Oscillation : The Dynamic Interaction of Visual and Vestibular Mechanisms, Aviation, Space, and Environmental Medicine, January, 1978, 340-345.

4. K.Taguchi, M.Kikukawa and T.Ishiyama, The Effects of Optokinetic Stimulation on the Head Movement, Equilibrium Res. Vol. 40(2), 1981, 242-250.( in Japanese ) 